\title{
Intracorneal Route of Administration
}

National Cancer Institute

\section{Source}

National Cancer Institute. Intracorneal Route of Administration. NCI Thesaurus. Code C38234.

Administration of a drug within the eye cornea. 